\title{
The efficacy of traditional Thai massage in decreasing spasticity in elderly stroke patients
}

\author{
This article was published in the following Dove Press journal: \\ Clinical Interventions in Aging \\ II August 2014 \\ Number of times this article has been viewed
}

\author{
Thanitta Thanakiatpinyo' \\ Supakij Suwannatrai ${ }^{2}$ \\ Ueamphon Suwannatrai ${ }^{2}$ \\ Phanitanong Khumkaew ${ }^{2}$ \\ Dokmai Wiwattamongkol ${ }^{2}$ \\ Manmas Vannabhum² \\ Somluck Pianmanakit ${ }^{1}$ \\ Vilai Kuptniratsaikul' \\ 'Department of Rehabilitation \\ Medicine, ${ }^{2}$ Center of Applied Thai \\ Traditional Medicine, Faculty of \\ Medicine Siriraj Hospital, Mahidol \\ University, Bangkok, Thailand
}

Purpose: To study the efficacy of traditional Thai massage (TTM) versus conventional physical therapy (PT) programs in treating muscle spasticity, functional ability, anxiety, depression, and quality of life (QoL) in Thai stroke patients.

Methods: This randomized controlled trial with a blinded assessor was carried out at the Department of Rehabilitation Medicine, Siriraj Hospital (Bangkok, Thailand). The study included 50 stroke (onset $\geq 3$ months) outpatients experiencing spasticity at the elbow or knee muscles at a grade of $\geq 1+$ on the modified Ashworth Scale who were $\geq 50$ years old and able to communicate. The subjects were randomly allocated to the treatment group receiving TTM (24 subjects) or the control group receiving the PT program (26 subjects). Both groups received treatment (either TTM or PT) twice a week for 6 weeks. Spasticity grade, functional ability, anxiety, depression, and QoL were measured at Week 0 and Week 6.

Results: At Week 6, the percentage of patients whose modified Ashworth Scale score had decreased by at least one grade was not statistically significant between the two groups. Both TTM and PT groups experienced a significant increase in functional ability and QoL, but no difference was found between the groups. Anxiety and depression scores showed a decreasing trend in the TTM group.

Conclusion: This preliminary report showed no evidence that TTM differed from the PT program in decreasing spasticity. However, both interventions may relieve spasticity, increase functional ability, and improve QoL after 6 weeks. Only TTM can decrease anxiety and depression scores. Further studies with adequate sample size are necessary.

Keywords: stroke, massage, spasticity, anxiety, depression

\section{Introduction}

Worldwide, stroke is one of the common ailments among the elderly. It is the thirdranking cause of death, and therefore affects the health care system in Thailand. ${ }^{1}$ One of the common consequences of stroke is spasticity. It is defined as a motor disorder that is characterized by a velocity-dependent increase in tonic stretch reflexes (muscle tone) with exaggerated tendon jerks, resulting from hyperexcitability of the stretch reflexes. ${ }^{2}$ The prevalence of post-stroke spasticity has ranged from $19 \%{ }^{3}$ to $39 \%{ }^{4}$ of stroke patients at 3 months and 12 months after experiencing stroke, respectively. In Thailand, the prevalence of post-stroke spasticity during rehabilitation was $41.6 \%{ }^{5}$ However, recent studies reported that the increased resistance of muscles with spasticity to passive movement is due not only to hyperexcitability reflexes, but also to altered properties of the muscle tissue. ${ }^{6,7}$ Spasticity can cause pain, abnormal posture, and joint contracture. It may interfere with functional recovery and the ability to perform daily activities, resulting in decreasing quality of life (QoL) and increasing caregiver burden.
Correspondence: Vilai Kuptniratsaiku Department of Rehabilitation Medicine, Faculty of Medicine Siriraj Hospital, Mahidol University, 2 Prannok Road, Bangkok 10700, Thailand

Tel +66 2419751 I

Fax +66 24 II 48I3

Email vilai.kup@mahidol.ac.th 
Currently, many methods for decreasing spasticity are available, including pharmacological and nonpharmacological treatments. The pharmacological therapies, such as oral anti-spastic drugs, ${ }^{8,9}$ botulinum toxin injection, ${ }^{9-11}$ phenol injections, ${ }^{9,12}$ alcohol injection, ${ }^{13}$ and intrathecal baclofen, ${ }^{14,15}$ usually focus on reducing reflex hyperexcitability. Nonpharmacological treatments, which aim to inhibit neural activity, reduce muscle stiffness, and improve the surrounding connective tissue, include heat modalities, cryotherapy, electrical stimulation, stretching, splinting, acupuncture, and massage. ${ }^{16,17}$

The mechanisms of massage on spasticity vary depending on technique. Goldberg et al found that petrissage massage above the lumbar area can reduce H-reflex amplitude in the calf muscles of people with traumatic spinal cord injury. ${ }^{18}$ This result reflects a reduction in motor neuron excitability. Deep massage can stretch the muscle that experiences spasticity and reset sarcomere lengths to a more optimal state. It helps to break down subcutaneous adhesions and prevent fibrosis, ${ }^{19}$ and may lead to improved sensory feedback from muscle spindle receptors. Moreover, the repetitive cutaneous stimulation provided by massage may reduce pain through the gate control theory. ${ }^{20}$

There have been a few study reports related to massage and spasticity. Unfortunately, these studies were reported in local languages. Only one randomized control trial was found, which reported the efficacy of rehabilitation exercise therapy in decreasing post-stroke hemiplegia limb muscle spasticity compared with traditional Chinese massage therapy. ${ }^{21}$ Their curative effects on patients were evaluated 4 weeks after treatment using the modified Ashworth Scale (MAS) and limb motor function integration scale, and by measuring the changes in activities of daily living. The researchers concluded, by examining total effective rates, that rehabilitation exercise therapy could improve muscle spasticity significantly more than traditional Chinese massage therapy. However, this study was published in Chinese. Thus, it cannot clearly be stated how the previous study measured the total effective rate and the details of the massage technique and dosage used.

Traditional Thai massage (TTM) is the most popular alternative medicine in Thailand. People have been acquainted with this massage for over a century. Currently, it is well recognized and regulated by the Thai government. Although massage is found worldwide in clinical practice, the scientific evidence that supports the effectiveness of massage in decreasing spasticity is limited. Therefore, a randomized controlled trial with a single-blinded assessor was performed to compare the effects of TTM and conventional physical therapy (PT) on spasticity, functional ability, anxiety, depression, and QoL in stroke patients.

\section{Methods}

The current study was a randomized trial. The study protocol was conducted in accordance with the ethical principles stated in the most recent version of the Declaration of Helsinki. After the study protocol was approved by the Institutional Review Board of Siriraj Hospital, stroke patients were recruited from the outpatient unit of the Department of Rehabilitation Medicine, Faculty of Medicine Siriraj Hospital (Bangkok, Thailand) from August 2011 through July 2013. Chronic stroke (onset $\geq 3$ months) patients with moderateto-severe spasticity of the elbow or knee in at least one limb ( rade $\geq 1+$ ), as evaluated by the MAS, who were aged $\geq 50$ years and able to communicate, were eligible to participate. Subjects who had contraindications for massage, eg, fever $38^{\circ} \mathrm{C}$ or greater, uncontrolled blood pressure, bleeding tendencies, taking anticoagulant drugs, unhealed fractures, contact dermatitis, skin infections, or severe osteoporosis, were excluded. Additionally, fixed joint contracture, a history of botulinum toxin injection within the last 6 months, a history of nerve block within the past year, severe dementia, or uncontrolled psychological disorders were excluded. The study would be terminated if severe adverse events occurred, including fractures or hematomas in the muscle or soft tissue of the patients.

After providing consent, subjects were randomly assigned to undergo TTM or the conventional PT program. A computergenerated randomization number was used, and the allocation codes were kept in opaque envelopes. An external investigator selected consecutive allocation envelopes for consecutive participants.

Patients who were randomized into the treatment group received court-type TTM, two nonconsecutive days per week for 6 weeks. Five certified personnel who performed massage for the duration of the study were standardized according to a Thai massage organization. Therapists performed the massage with the same procedure over the hemiplegic side for 1 hour per session. The basic massage lines and major signal points were the main massage treatment using only thumb and hand pressing at the point without traction or stretching. The basic massage lines were pressed for 10 seconds and the major signal points were pressed for 30 seconds per point. Massage points included in this study were located in the leg, back, abdomen, arm, shoulder, and neck regions. The details of the massage protocol are presented in Table S1. 
The PT group received a range of motion exercises for paralyzed limbs, strengthening exercises for the sound limbs, balance exercises for sitting and standing, and ambulation training in those who had potential for walking. The program was administered by a trained physical therapist for 1-hour sessions, two times a week for 6 weeks.

Regarding the primary outcome, the percentage of patients who improved after treatment, ie, patients whose MAS decreased by at least one grade after treatment, was measured. The MAS is a common clinical test used to determine spasticity, with ratings from zero (no increase in muscle tone) to four (the affected part is rigid in flexion or extension). ${ }^{22}$ For secondary outcomes, the Barthel Index (BI) was used to measure functional abilities in self-care and mobility. ${ }^{23}$ Possible scores range from $0-20$, with a higher score meaning better functions. To measure the patients' psychological status, the Thai version of the Hospital Anxiety and Depression Scale (HADS) was used to screen for anxiety and depression. ${ }^{24}$ The scores for each subscale (anxiety and depression) range from 0-21 with scores of eleven or greater indicating the presence of a disorder. ${ }^{24-26}$ Additionally, the Pictorial Thai QoL Test was used to evaluate QoL. ${ }^{27}$ It is composed of six dimensions: physical, cognitive, affective, social function, economics, and self-esteem. Scores range from $0-72$, where a higher score represents a better quality of life. The adverse events that occurred in both groups were also recorded. At the end of the study, the patients were asked about feelings of spasticity and the use of limbs' functions as subjective outcomes. Satisfaction level was also evaluated among both groups.

\section{Statistical analysis}

The sample size of 186 patients (93 in each group) provides a power of $80 \%$ to detect differences in the primary outcome (ie, the percentage of patients whose spasticity decreased by at least one grade after treatment) between a PT group of $30 \%$ and a TTM group of $50 \%$, at a $5 \%$ two-sided significance level. ${ }^{28}$

Demographic and baseline characteristics are presented as descriptive statistics in Table 1. Unpaired $t$-test was used to compare continuous data including age, BI, HADS, and QoL scores. Chi-square and Fisher's exact tests were used to compare categorical data. Multiple logistic regression was used to adjust for age and sex for comparing the primary outcome. Mean changes in the scores of the BI, HADS, and Pictorial Thai QoL at baseline, compared with 6-week scores, were analyzed using paired $t$-tests, and the mean differences in those scores were compared between groups by using unpaired $t$-tests. The number of adverse events and patients' assessment of outcomes were compared using the chi-square test and Fisher's exact test. A $P$-value of less than 0.05 was considered statistically significant. For the primary outcome,

Table I Demographic and baseline characteristics

\begin{tabular}{|c|c|c|c|}
\hline Demographic and baseline characteristics & $\begin{array}{l}\text { TTM group } \\
(\mathrm{n}=\mathbf{2 4 )}\end{array}$ & $\begin{array}{l}\text { PT group } \\
(n=26)\end{array}$ & $P$-value ${ }^{\#}$ \\
\hline Sex: male & $22(91.7 \%)$ & $15(57.7 \%)$ & $0.006 *$ \\
\hline Age (in years) & $60.0 \pm 6.9$ & $65.8 \pm 8.1$ & $0.010^{*}$ \\
\hline Education: primary school & $7(29.2 \%)$ & $5(19.2 \%)$ & 0.411 \\
\hline \multicolumn{4}{|l|}{ Underlying } \\
\hline Diabetes mellitus type 2 & $9(37.5 \%)$ & II (42.3\%) & 0.729 \\
\hline Hypertension & $23(95.8 \%)$ & $23(88.5 \%)$ & 0.611 \\
\hline Dyslipidemia & $19(79.2 \%)$ & $19(73.1 \%)$ & 0.614 \\
\hline Cardiac disease & $2(8.3 \%)$ & $4(15.4 \%)$ & 0.669 \\
\hline Onset: first attack & $18(75.0 \%)$ & $18(69.2 \%)$ & 0.650 \\
\hline Pathology: infarction & $12(50.0 \%)$ & $15(57.7 \%)$ & 0.585 \\
\hline Surgery: yes & $4(16.7 \%)$ & $8(30.8 \%)$ & 0.243 \\
\hline Muscles with maximum grading ${ }^{\dagger}$ & & & 0.296 \\
\hline $\mathrm{I}+$ & $13(54.1 \%)$ & $19(73.1 \%)$ & \\
\hline 2 & $10(41.7 \%)$ & 5 (19.2\%) & \\
\hline 3 & I (4.2\%) & $2(7.7 \%)$ & \\
\hline Barthel Index (0-20) & $16.4 \pm 4.3$ & $10.9 \pm 6.6$ & $0.001 *$ \\
\hline HADS: anxiety score (0-2I) & $4.8 \pm 3.9$ & $4.1 \pm 3.1$ & 0.529 \\
\hline HADS: depression score $(0-2 I)$ & $5.1 \pm 4.3$ & $3.9 \pm 3.5$ & 0.284 \\
\hline Pictorial Quality of Life score (0-72) & $4 I .1 \pm 15.6$ & $42.8 \pm 13.7$ & 0.691 \\
\hline
\end{tabular}

Notes: \#npaired $t$-test for continuous data, chi-square test, and Fisher's exact test for categorical data. 'Muscles with maximum grading, evaluated using the modified Ashworth Scale. * Statistically significant at $P<0.05$.

Abbreviations: HADS, Hospital Anxiety and Depression Scale; PT, physical therapy; TTM, traditional Thai massage. 
intention-to-treat (ITT) analysis, with the last observation carried forward method, was performed in addition to per protocol (PP) analysis.

\section{Results}

A total of 220 stroke patients were enrolled in the study, but 170 subjects were screened out owing to their spasticity grade (MAS) being $<1+$ (69 patients), inability to communicate (38 patients), complaints of inconvenience (43 patients), or refusals to change their treatment program (20 patients). Only 50 patients were randomized to receive TTM (24 patients) or the PT program (26 patients) twice weekly for the 6-week duration. At the end of study, five patients in the PT group were lost to follow-up owing to inconvenience (four patients) and having repeat hemorrhagic strokes (one patient), as shown in Figure 1. The demographic data of the 50 subjects in the TTM and PT groups did not differ except for sex, age, and BI scores (Table 1). Patients in the TTM group were predominantly male ( $92 \%$ versus $58 \%$ ), younger in age (60 years versus 66 years), and had higher BI scores (16.4 versus 10.9) compared with the PT group. A muscle with maximal grading measured by MAS was not different between the groups. Anxiety, depression, and QoL scores did not differ between the groups at baseline.
The main outcome was the percentage of subjects whose MAS score decreased by at least one grade after treatment. The TTM group had a higher percentage of subjects whose spasticity improved by at least one grade than the PT group in both types of analysis $(70.8 \%$ versus $61.9 \%$ in PP analysis; $70.8 \%$ versus $50.0 \%$ in ITT analysis). However, there was no statistically significant difference between the two groups, regardless of whether it was analyzed by PP analysis $(P=0.286)$ or ITT analysis $(P=0.058)$ in muscles with maximal grade (Tables 2 and 3 , respectively).

Figure 2 shows that, for secondary outcomes, BI scores increased significantly at Week 6 compared with baseline in both groups $(P=0.008$ and $P=0.025$ in TTM and PT groups, respectively), but no difference was found between the groups $(P=0.805)$. Figure 3 illustrates the psychological aspects; anxiety scores in the TTM group had decreased at Week 6 compared with baseline scores $(P=0.083)$, while the anxiety scores in the PT group had increased at Week 6 $(P=0.624)$. However, there was no difference in the scores when the two groups were compared $(P=0.144)$. Figure 4 shows the depression scores after treatment. The depression scores in the TTM group had decreased at Week 6 compared with baseline scores but were not significantly

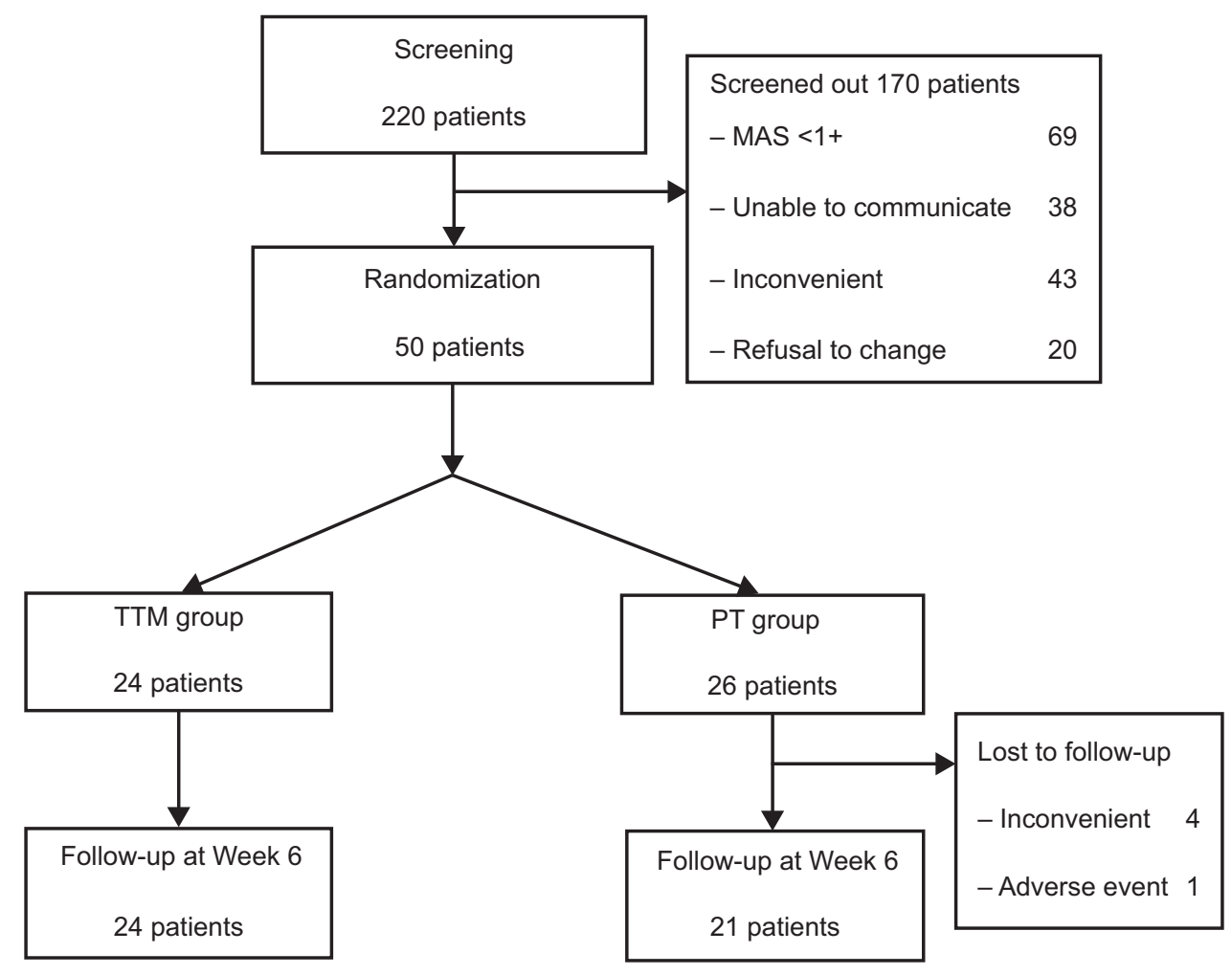

Figure I Flow diagram of the study.

Abbreviations: MAS, modified Ashworth Scale; PT, physical therapy; TTM, traditional Thai massage. 
Table 2 Number and percentage of subjects whose spasticity decreased by at least one grade between baseline and after treatment among the two groups, using per protocol analysis

\begin{tabular}{|c|c|c|c|c|c|c|}
\hline \multirow[t]{3}{*}{ Baseline } & \multicolumn{6}{|c|}{ After treatment } \\
\hline & \multicolumn{3}{|c|}{ TTM group $(n=24)$} & \multicolumn{3}{|c|}{ PT group $(n=2 I)$} \\
\hline & Grade $\leq I$ & Grade I+ & Grade 2 & Grade $\leq I$ & Grade I+ & Grade 2 \\
\hline Grade I+ & $9(37.5 \%)$ & $4(16.7 \%)$ & 0 & $9(42.8 \%)$ & $6(28.6 \%)$ & 0 \\
\hline Grade 2 & $5(20.8 \%)$ & $2(8.3 \%)$ & $3(12.5 \%)$ & I (4.8\%) & I (4.8\%) & $2(9.5 \%)$ \\
\hline Grade 3 & 0 & I (4.2\%) & 0 & 0 & 0 & $2(9.5 \%)$ \\
\hline
\end{tabular}

Notes: Improvement is defined as the spasticity of muscles with maximum grading, decreased by at least one grade. $P=0.286$ after adjusting for age and sex using multiple logistic regression analysis.

Abbreviations: PT, physical therapy; TTM, traditional Thai massage.

different $(P=0.195)$, whereas depression scores increased in the PT group $(P=0.747)$. No difference was found between the groups $(P=0.313)$.

Figure 5 presents the QoL of the TTM and PT groups at Week 0 and Week 6. At the end of study, the total QoL scores of both groups had increased significantly when compared with baseline scores ( $P=0.041$ in TTM; $P=0.001$ in PT groups). However, there was no significant difference between the groups ( $P=0.715$ ).

Table 4 compares patients' assessments of their outcomes by group. The number of patients in the TTM group who reported that their feelings of spasticity had decreased was greater than those who reported the same in the PT group (79.2\% versus $52.4 \% ; P=0.057)$. However, the functions of their limbs improved only $37 \%-38 \%$ with no difference between the groups $(P=0.967)$. Concerning patient satisfaction, $90.5 \%$ of the patients were satisfied with the PT program compared with $75 \%$ of the massage patients. Again, there was no significant difference between the two groups $(P=0.427)$. The number of adverse events that occurred during the study did not differ between the groups $(P=0.807)$, as shown in Table 5 . There were more patients who experienced adverse events of muscle stiffness, muscle pain, and muscle soreness in the TTM group compared with those in the PT group. However, no significant difference between the groups was found.

\section{Discussion}

TTM is one of the methods of alternative medicine that is easily accessible in Thailand. People choose massage with the aim of relaxing their muscles. It is very popular among Thai people and patients alike. Studies examining whether TTM was effective in decreasing muscle spasticity in stroke patients were scarce. The current study revealed that the percentage of subjects whose MAS scores had decreased by at least one grade after treatment in the TTM group seemed to be greater than that of the PT program group. After using ITT analysis, the results displayed more significant differences than those from the PP analysis; however, no significant difference was found in both analyses. Additionally, the number of patients in the TTM group who reported that their feelings of spasticity decreased was greater than that of the PT group. This subjective outcome was consistent with the number of patients whose MAS (ie, an objective outcome) had decreased by at least one grade. However, these findings were not in line with Chen and Li's study. They reported that rehabilitation exercise therapy can improve muscle spasticity in post-stroke hemiplegia significantly more than traditional Chinese massage therapy. ${ }^{21}$ This might be due to the different style, as well as dosage, of massage.

Although both TTM and PT programs increased the BI score by the conclusion of the study, there was no difference in the BI score between the two groups. It was assumed that

Table 3 Number and percentage of subjects whose spasticity decreased by at least one grade between baseline and after treatment among the two groups, using intention-to-treat analysis with the last observation carried forward method

\begin{tabular}{|c|c|c|c|c|c|c|}
\hline \multirow[t]{3}{*}{ Baseline } & \multicolumn{6}{|c|}{ After treatment } \\
\hline & \multicolumn{3}{|c|}{ TTM group $(n=24)$} & \multicolumn{3}{|c|}{ PT group $(n=26)$} \\
\hline & Grade $\leq \mathbf{I}$ & Grade I+ & Grade 2 & Grade $\leq \mathbf{I}$ & Grade I+ & Grade 2 \\
\hline Grade I+ & 9 (37.5\%) & $4(16.7 \%)$ & 0 & 9 (34.6\%) & $10(38.5 \%)$ & 0 \\
\hline Grade 2 & $5(20.8 \%)$ & $2(8.3 \%)$ & $3(12.5 \%)$ & I (3.8\%) & I (3.8\%) & 3 (11.6\%) \\
\hline Grade 3 & 0 & I (4.2\%) & 0 & 0 & 0 & $2(7.7 \%)$ \\
\hline
\end{tabular}

Notes: Improvement is defined as the spasticity of muscles with maximum grading, decreased by at least one grade. $P=0.058$ after adjusting for age and sex using multiple logistic regression analysis.

Abbreviations: PT, physical therapy; TTM, traditional Thai massage. 


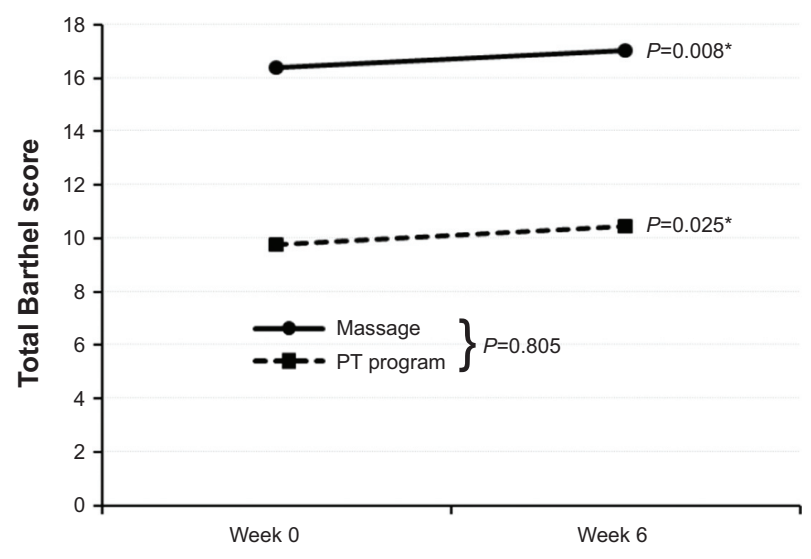

Figure 2 Functional ability evaluated using the Barthel Index at Week 0 and Week 6. Note: *Statistically significant at $P<0.05$.

Abbreviation: PT, physical therapy.

the decrease in spasticity in both groups may result in subjects using their affected limbs more easily and causing functional improvement after treatment. Additionally, the ability to perform daily activities can improve through spontaneous motor recovery in subacute stroke patients and functional adaptation after PT program. ${ }^{29}$

A decreasing trend in anxiety and depression scores at Week 6 was only found in the TTM group, whereas those in the PT group seemed to exhibit an increasing trend instead. This means that only the TTM group reported trends of improvement in anxiety and depression posttreatment. The reasons for the differences in psychological outcomes between groups are possible from enhancing the parasympathetic nervous system ${ }^{30}$ and relieving muscle tension in the TTM group. ${ }^{31}$ These results were consistent with a meta-analysis of massage therapy research by Moyer et al who reported that multiple doses of massage therapy can significantly reduce the traits of anxiety and depression. ${ }^{32}$

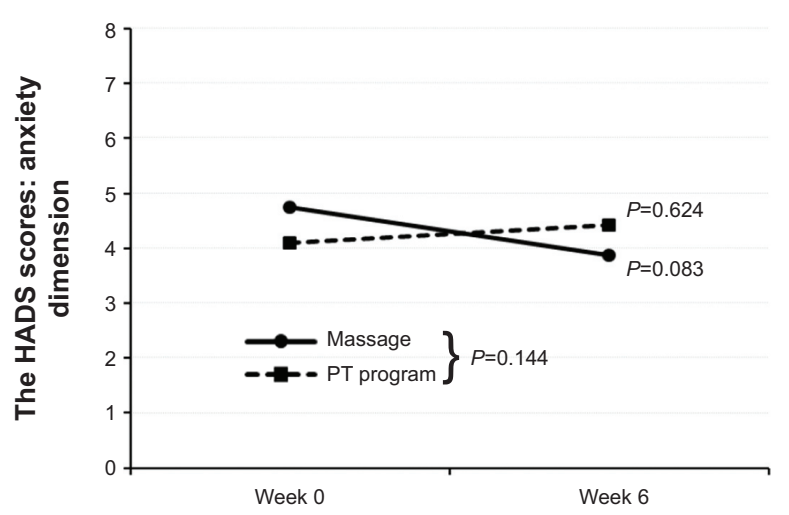

Figure 3 Anxiety scores at Week 0 and Week 6.

Abbreviations: HADS, Hospital Anxiety and Depression Scale; PT, physical therapy.

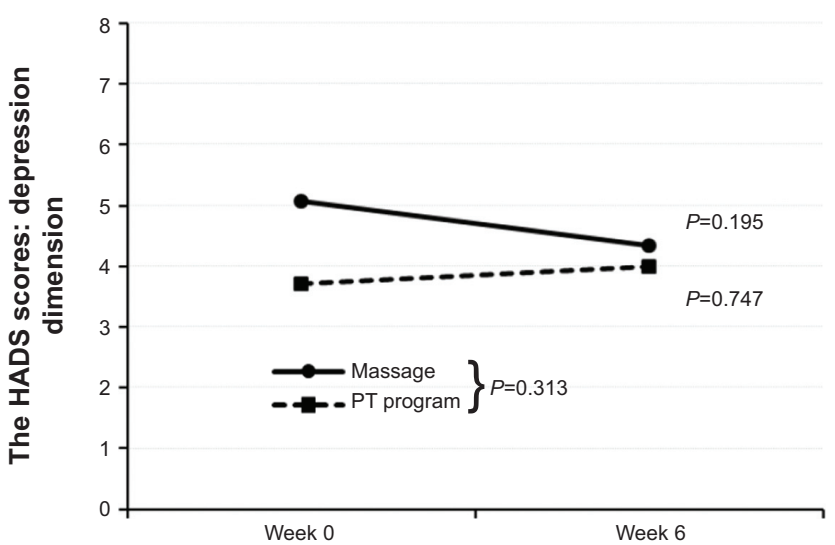

Figure 4 Depression scores at Week 0 and Week 6.

Abbreviations: HADS, Hospital Anxiety and Depression Scale; PT, physical therapy.

Moreover, QoL scores in both the TTM and PT group increased between baseline and Week 6, with no difference between the two groups. This means that both TTM and PT treatments may improve QoL in stroke patients.

Ninety percent of the patients in the PT group were satisfied with the treatment program, compared with only $75 \%$ of the TTM group reporting satisfaction. The reason why patients have been less satisfied with TTM may be due to the number of adverse events that occurred in the massage group, especially muscle stiffness, muscle pain, and muscle soreness. This finding was consistent with a qualitative study by van der Riet et al on TTM used in stroke patients. ${ }^{33}$ They reported that some stroke patients experienced pain after receiving massages, and the younger patients preferred to exercise rather than to receive massages. Though massage seems to be effective in decreasing spasticity in stroke patients, medical personnel should recognize that pressure during massage could adversely affect muscles. Therefore, standardized techniques of massage, in addition

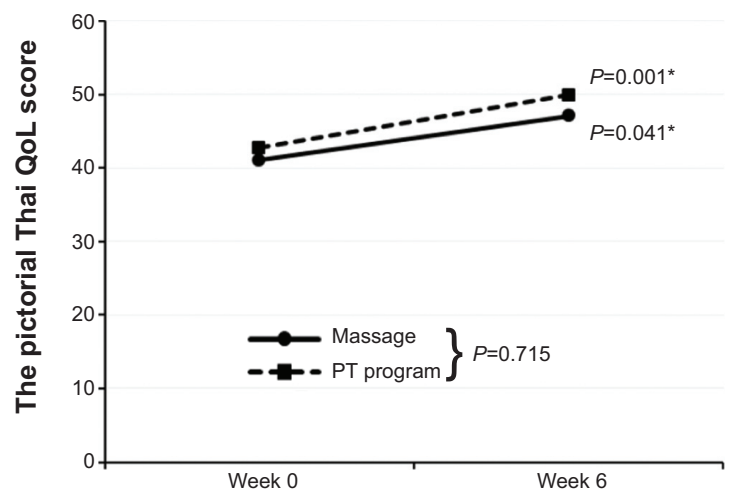

Figure 5 Quality of life scores at Week 0 and Week 6.

Note: *Statistically significant at $P<0.05$.

Abbreviations: PT, physical therapy; QoL, quality of life. 
Table 4 Patients' assessment of outcomes

\begin{tabular}{|c|c|c|c|}
\hline Patients' assessment & $\begin{array}{l}\text { TTM group } \\
(n=24)\end{array}$ & $\begin{array}{l}\text { PT group } \\
(n=2 I)\end{array}$ & P-value $\#$ \\
\hline \multicolumn{4}{|l|}{ Feelings of spasticity } \\
\hline Decreased & 19 (79.2\%) & II (52.4\%) & 0.057 \\
\hline Indifferent & $5(20.8 \%)$ & $10(47.6 \%)$ & \\
\hline \multicolumn{4}{|l|}{ Use of limbs (functions) } \\
\hline Improved & $9(37.5 \%)$ & $8(38.1 \%)$ & 0.967 \\
\hline Indifferent & $15(62.5 \%)$ & $13(61.9 \%)$ & \\
\hline \multicolumn{4}{|l|}{ Satisfaction } \\
\hline Satisfied & 18 (75.0\%) & $19(90.5 \%)$ & 0.427 \\
\hline Indifferent & $4(16.7 \%)$ & $2(9.5 \%)$ & \\
\hline Dissatisfied & $2(8.3 \%)$ & 0 & \\
\hline
\end{tabular}

Note: \#Chi-square test, Fisher's exact test.

Abbreviations: PT, physical therapy; TTM, traditional Thai massage.

to the development of equipment for measuring pressure, are necessary.

\section{Study limitations}

The main limitation of this study was the small sample size. A large number of patients were excluded for reasons such as communication difficulties, spasticity that was too mild, wishing to avoid inconvenience, and refusal to change their current treatment program. In addition, this study only recruited outpatients. Some patients could not visit the hospital without assistance, so they declined to join the study. Moreover, there were slight differences in the demographic characteristics between the two groups. These differences could influence the results of study. For example, a low BI at baseline has been suggested as one of the predictors for the presence of severe spasticity. Thus, subjects who had a low BI score in the PT group may have a higher chance of developing spasticity ${ }^{34}$ and less potential for recovery. ${ }^{35} \mathrm{On}$ the other hand, the $\mathrm{BI}$ is not sensitive for detecting change in stroke patients with a high BI score at baseline due to its ceiling effect. ${ }^{36}$ However, these variables were adjusted during analysis of the results. Finally, this study did not evaluate the long-term effect of massage on spasticity.

Table 5 Number of adverse events that occurred during the study by group

\begin{tabular}{|c|c|c|c|}
\hline Adverse events & $\begin{array}{l}\text { TTM group } \\
(n=24)\end{array}$ & $\begin{array}{l}\text { PT group } \\
(n=2 I)\end{array}$ & $P$-value ${ }^{\#}$ \\
\hline Number of adverse events & 10 (4I.7\%) & $8(38.1 \%)$ & 0.807 \\
\hline Muscle stiffness & $8(33.3 \%)$ & $5(23.8 \%)$ & 0.482 \\
\hline Muscle pain & 7 (29.2\%) & $5(23.8 \%)$ & 0.685 \\
\hline Muscle soreness & $4(16.7 \%)$ & I (4.8\%) & 0.352 \\
\hline Ecchymoses & I (4.2\%) & I (4.8\%) & 1.000 \\
\hline Joint swelling & I (4.2\%) & 0 & 1.000 \\
\hline
\end{tabular}

Note: "Chi-square test, Fisher's exact test.

Abbreviations: PT, physical therapy; TTM, traditional Thai massage.
Further studies with adequate sample size and long term follow-up are suggested.

\section{Conclusion}

This preliminary study could not demonstrate that TTM differs from conventional PT programs in decreasing spasticity or improving limb functions. Although no statistical significance was found, there were trends in decreasing spasticity, anxiety, and depression scores in the TTM group compared with the PT group. Massage may be an alternative method for stroke patients to decrease spasticity, improve psychological conditions and QoL. Further studies employing an adequate sample size and evaluating the long-term effect of massage are needed.

\section{Acknowledgments}

The authors would like to thank Mr Suthiphol Udompunturuk and Ms Julaporn Poolium for statistical analyses, Assistant Professor Somkiat Sangwatanaroj for translating Chinese research into Thai language, and Professor Manee Rattanachaiyanont for reviewing the manuscript.

This study was supported by the National Research Council of Thailand (NRCT). The trial registration number is NCT01448980.

\section{Disclosure}

The authors report no conflicts of interest in this work.

\section{References}

1. Bureau of Health Policy and Strategy Ministry of Public Health. [Public Health Statistics 2010]. Bangkok: Thailand Ministry of Public Health; 2010. Available from: http://bps.ops.moph.go.th/Healthinformation/ statistic53/statistic53.pdf. Accessed November 20, 2013. Thai.

2. Lance JW. The control of muscle tone, reflexes, and movement: Robert Wartenberg Lecture. Neurology. 1980;30(12):1303-1313.

3. Sommerfeld DK, Eek EU, Svensson AK, Holmqvist LW, von Arbin MH. Spasticity after stroke: its occurrence and association with motor impairments and activity limitations. Stroke. 2004;35(1):134-139.

4. Watkins CL, Leathley MJ, Gregson JM, Moore AP, Smith TL, Sharma AK. Prevalence of spasticity post stroke. Clin Rehabil. 2002;16(5): 515-522.

5. Dajpratham P, Kuptniratsaikul V, Kovindha A, Kuptniratsaikul P, Dejnuntarat K. Prevalence and management of poststroke spasticity in Thai stroke patients: a multicenter study. J Med Assoc Thai. 2009;92(10): 1354-1360.

6. Lieber RL, Steinman S, Barash IA, Chambers H. Structural and functional changes in spastic skeletal muscle. Muscle Nerve. 2004;29(5): 615-627.

7. Foran JR, Steinman S, Barash I, Chambers HG, Lieber RL. Structural and mechanical alterations in spastic skeletal muscle. Dev Med Child Neurol. 2005;47(10):713-717.

8. Abbruzzese G. The medical management of spasticity. Eur J Neurol. 2002;9(Suppl 1):S30-S34.

9. Gallichio JE. Pharmacological management of spasticity following stroke. Phys Ther. 2004;84(10):973-981. 
10. Turkel CC, Bowen B, Liu J, Brin MF. Pooled analysis of the safety of botulinum toxin type A in the treatment of poststroke spasticity. Arch Phys Med Rehabil. 2006;87(6):786-792.

11. Ozcakir S, Sivrioglu K. Botulinum toxin in poststroke spasticity. Clin Med Res. 2007;5(2):132-138.

12. McCrea PH, Eng JJ, Willms R. Phenol reduces hypertonia and enhances strength: a longitudinal case study. Neurorehabil Neural Repair. 2004; 18(2):112-116.

13. Jang SH, Ahn SH, Park SM, Kim SH, Lee KH, Lee ZI. Alcohol neurolysis of tibial nerve motor branches to the gastrocnemius muscle to treat ankle spasticity in patients with hemiplegic stroke. Arch Phys Med Rehabil. 2004;85(3):506-508.

14. Meythaler JM, Guin-Renfroe S, Brunner RC, Hadley MN. Intrathecal baclofen for spastic hypertonia from stroke. Stroke. 2001;32(9): 2099-2109.

15. Francisco GE, Boake C. Improvement in walking speed in poststroke spastic hemiplegia after intrathecal baclofen therapy: a preliminary study. Arch Phys Med Rehabil. 2003;84(8):1194-1199.

16. Nance PW, Meythaler JM. Spasticity management. In: Braddom RL, editor. Physical Medicine and Rehabilitation. 3rd ed. Philadelphia: Saunders; 2007:661

17. Watanabe T. The role of therapy in spasticity management. Am J Phys Med Rehabil. 2004;83(10 Suppl):S45-S49.

18. Goldberg J, Seaborne DE, Sullivan SJ, Leduc BE. The effect of therapeutic massage on H-reflex amplitude in persons with a spinal cord injury. Phys Ther. 1994;74(8):728-737.

19. Donnelly CJ, Wilton J. The effect of massage to scars on active range of motion and skin mobility. Hand Ther. 2002;7(1):5-11.

20. Gracies JM. Physical modalities other than stretch in spastic hypertonia. Phys Med Rehabil Clin N Am. 2001;12(4):769-792.

21. Chen Q, Li L. Observation on curative effect of rehabilitation exercise therapy to treat patients with post-stroke hemiplegia and muscles spasm. Chinese Nursing Research. 2010;24(1):14-16.

22. Bohannon RW, Smith MB. Interrater reliability of a modified Ashworth scale of muscle spasticity. Phys Ther. 1987;67(2):206-207.

23. Mahoney FI, Barthel DW. Functional evaluation: the Barthel Index. Md State Med J. 1965;14:61-65.
24. Nilchaikovit T, Lortrakul M, Phisansuthideth U. Development of Thai version of Hospital Anxiety and Depression Scale in cancer patients. J Psychiatr Assoc Thai. 1996;41(1):18-30.

25. Zigmond AS, Snaith RP. The Hospital Anxiety and Depression Scale. Acta Psychiatr Scand. 1983;67(6):361-370.

26. Bjelland I, Dahl AA, Haug TT, Neckelmann D. The validity of the Hospital Anxiety and Depression Scale: an updated literature review. J Psychosom Res. 2002;52(2):69-77.

27. Phattharayuttawat S, Ngamthipwatthana T, Pitiyawaranun B. The development of the Pictorial Thai Quality of Life. J Med Assoc Thai. 2005;88(11):1605-1618.

28. Barros Galvao SC, Borba Costa dos Santos R, Borba dos Santos P, Cabral ME, Monte-Silva K. Efficacy of coupling repetitive transcranial magnetic stimulation and physical therapy to reduce upper-limb spasticity in patients with stroke: a randomized controlled trial. Arch Phys Med Rehabil. 2014;95(2):222-229.

29. Kotila M, Waltimo O, Niemi ML, Laaksonen R, Lempinen M. The profile of recovery from stroke and factors influencing outcome. Stroke. 1984;15(6):1039-1044.

30. Diego MA, Field T. Moderate pressure massage elicits a parasympathetic nervous system response. Int J Neurosci. 2009;119(5):630-638.

31. Jorm AF, Christensen H, Griffiths KM, Parslow RA, Rodgers B, Blewitt KA. Effectiveness of complementary and self-help treatments for anxiety disorders. Med J Aust. 2004;181(7 Suppl):S29-S46.

32. Moyer CA, Rounds J, Hannum JW. A Meta-analysis of massage therapy research. Psychol Bull. 2004;130(1):3-18.

33. van der Riet P, Dedkhard S, Srithong K. Complementary therapies in rehabilitation: stroke patients' narratives. Part 2. J Clin Nurs. 2012; 21(5-6):668-676.

34. Wissel J, Schelosky LD, Scott J, Christe W, Faiss JH, Mueller J. Early development of spasticity following stroke: a prospective, observational trial. J Neurol. 2010;257(7):1067-1072.

35. Coupar F, Pollock A, Rowe P, Weir C, Langhorne P. Predictors of upper limb recovery after stroke: a systematic review and meta-analysis. Clin Rehabil. 2012;26(4):291-313.

36. Duncan PW, Samsa GP, Weinberger M, et al. Health status of individuals with mild stroke. Stroke. 1997;28(4):740-745. 


\section{Supplementary material}

Table SI Step, area, and duration of traditional Thai massage protocol

\begin{tabular}{lll}
\hline Step & Areas & Duration \\
\hline I & BML-leg (beginning from lower leg to upper leg then return to lower leg) and MaSP-ankle (press at anterior of ankle joint) & 6 minutes \\
2 & BML-back (along erector spinae muscle from L5 to C7) & 9 minutes \\
3 & BML and MaSP-lateral side of leg (beginning from gluteal area to lateral side of upper and lower leg) & 5 minutes \\
4 & BML and MaSP-medial side of leg (beginning from medial side of upper leg to lower leg) & 5 minutes \\
5 & BML-abdomen (around abdomen) & 10 minutes \\
6 & BML and MaSP-medial side of arm (beginning from middle of medial side of upper arm to anterior of wrist) & 5 minutes \\
7 & BML and MaSP-lateral side of arm (beginning from middle of lateral side of upper arm to middle of lower arm) & 5 minutes \\
8 & BML-shoulder (upper part of back at posterior shoulder) & 5 minutes \\
9 & BML-neck (posterior of neck) & 5 minutes \\
10 & MaSP-shoulder (lateral side of lower neck and posterior of shoulder) & 5 minutes \\
\hline
\end{tabular}

Notes: In steps I, 5, 6, and 7, the subject was lying supine. In steps 2-4, the subject was lying on their non-affected side. In steps 8-10, the subject was in a sitting position. Abbreviations: BML, basic massage lines; MaSP, major signal points.

\section{Publish your work in this journal}

Clinical Interventions in Aging is an international, peer-reviewed journal focusing on evidence-based reports on the value or lack thereof of treatments intended to prevent or delay the onset of maladaptive correlates of aging in human beings. This journal is indexed on PubMed Central, MedLine,
CAS, Scopus and the Elsevier Bibliographic databases. The manuscript management system is completely online and includes a very quick and fair peer-review system, which is all easy to use. Visit http://www.dovepress. com/testimonials.php to read real quotes from published authors. 